Bull. Mater. Sci., Vol. 22, No. 3, May 1999, pp. 329-333. (C) Indian Academy of Sciences.

\title{
Mono-dispersed single-wall carbon nanotubes formed in channels of zeolite crystal: Production, optical and electrical transport properties
}

\author{
Z K TANG*, H D SUN, J WANG, J CHEN ${ }^{\dagger}$ and G $\mathbf{L I}^{\dagger}$ \\ Physics Department, Hong Kong University of Science and Technology, Clear Water Bay, Kowloon, \\ Hong Kong, China \\ 'Department of Chemistry, Jilin University, 119 Jiefang Road, Changchun 130023, China
}

\begin{abstract}
Carbon nanotube materials can now be produced in macroscopic quantities. However, the raw material has a disordered structure and unsorted size, which restrict investigations of both the properties and applications of the nanotubes. In this paper, an alternative approach to the synthesis of mono-sized and parallel-aligned single wall carbon nanotubes (SWCNs) is reported. The SWCNs are formed in $1 \mathrm{~nm}$-sized channels of aluminophosphate zeolite crystallites by pyrolysis of tripropylamine molecules. As verified by tunnel electron microscopy and micro-Raman scattering, the SWNT is of zigzag structure. Electrical transport properties of the SWNT are measured in the temperature range of $0.3 \mathrm{~K} \sim 300 \mathrm{~K}$. The temperature-dependent de conductivity shows that the SWNT is an intrinsic semiconductor with a narrow band-gap of $52 \mathrm{meV}$. The well-aligned and mono-sized SWCNs allow us to make more controlled characterization as well as open a door to potential nano-technological application for the novel electronic nanotube system.
\end{abstract}

Keywords. Carbon nanotubes; zeolite crystal.

\section{Introduction}

Carbon nanotubes were discovered in 1991 from the cathode product by carbon-arc discharge method similar to that used for fullerene preparation (Iijima 1991). This discovery triggered extensive theoretical and experimental researches, and led to a rapidly developing research field. The nanotube system offers new prospects in fundamental as well as nano-technological applications. Besides the carbon-arc discharge technique, carbon nanotubes can now also be produced using laser evaporation (Guo et al 1995), and catalysis of hydrocarbons (Amelincks et al 1994; Kukovitsukii 1997). In the earlier works, the nanotube products found mostly included fragments of graphene sheets, carbon clusters and amorphous carbons. Recently, purification process to obtain nanotubes out of the mixture carbon specimens was gradually established. An important recent advance in carbon nanotube science is the synthesis of single-walled carbon nanotubes (SWCNs) in high yield using laser ablation (Guo $e t$ al 1995) and electric arc technique (Journet et al 1997), in each case a small amount of transition metal is added to the carbon target as a catalyst. The ability of SWCN synthesis brings the experimental situation much closer to that of the theoretical models. However, it remains to be a challenge to produce mono-sized SWCNs with well-defined chirality.

A SWCN is formed from a single layer of graphene sheet. The surface of the cylinder has a honeycomb-lattice

*Author for correspondence pattern, just as in a two-dimensional graphite plane. From theoretical point of view, it is interesting as the embodiment of a one-dimensional periodic structure along the tube axis. Depending on the rolling direction of the graphene sheet, a carbon nanotube has three possible structures: zigzag, armchair and chiral, which can uniquely be represented by a so-called roll-up vector $(n, m)$ (Dresselhaus et al 1995). For the zigzag structure $m=0$, and for the armchair structure $m=n$. The electronic property of a carbon nanotube is expected to be purely dependent on its geometrical structure (chirality and tube size). An armchair nanotube is metal, while a zigzag nanotube is a semiconductor with a narrow band gap (when $n$ is the multiply of three) or with a moderate band-gap (for other $n$ ) (Blase et al 1994; Mintmire and White 1995). Independent of whether the nanotubes are conducting or semiconducting, the energy bands are expected to show one-dimensional characteristic densityof-state with a $\left(E-E_{\mathrm{i}}\right)^{-1 / 2}$ type singularity at its band maximum energy $E_{\mathrm{i}}$ (Dresselhaus et al 1996). However, experimental measurements to test these remarkable expectations of the electronic structure are not easy because of the difficulty in fabricating mono-sized carbon nanotubes with well-defined structural symmetry. Another technical challenge is to make a good electric electrode to such a small tube. Up to now, the experimental studies have been mainly limited to nanotube bundles (Thess et al 1996; Lee et al 1997) and individual multi-walled carbon nanotubes (Ebbesen et al 1996; Langer et al 1996), which only qualitatively show semiconductor or metal properties of the nanotubes. Recently, measurements 
using two-probe configuration showed that individual SWCNs behave as molecular wires, exhibiting quantum effects at low temperature (Bockrath et al 1997; Tans et al 1997). For more controlled characterization of these novel electronic properties, separated, aligned and monosized nanotubes with a well-defined chirality are essential.

In this paper, we report an alternative approach to the synthesis of mono-sized and parallel-aligned SWCNs in channels of micro-porous zeolite crystal by pyrolysis of tripropylamine (TPA). The nanotube structure is confirmed using the transmission electron microscopic (TEM) observation, and characterized using Raman scattering. Optical and electrical transport properties are studied for the mono-sized carbon nanotube system. The fabrication of mono-dispersed SWCNs in three-dimensional ordered and nano-sized channels represents an important step towards the development and applications of carbon nanotube materials.

\section{Experimental}

Microporous aluminophosphate $\mathrm{AlPO}_{4}-5$ crystals (AFI) with elongated hexagonal prisms of dimensions $110 \mu \mathrm{m}$ in cross-section diameter and $300 \mu \mathrm{m}$ in length were used as the hosts to encapsulate the SWCNs. The framework of the AFI crystal was constructed of alternative tetrahedra of $\left(\mathrm{AlO}_{4}\right)^{-}$and $\left(\mathrm{PO}_{4}\right)^{+}$, which form parallel open channels, packed in hexagonal structure. The

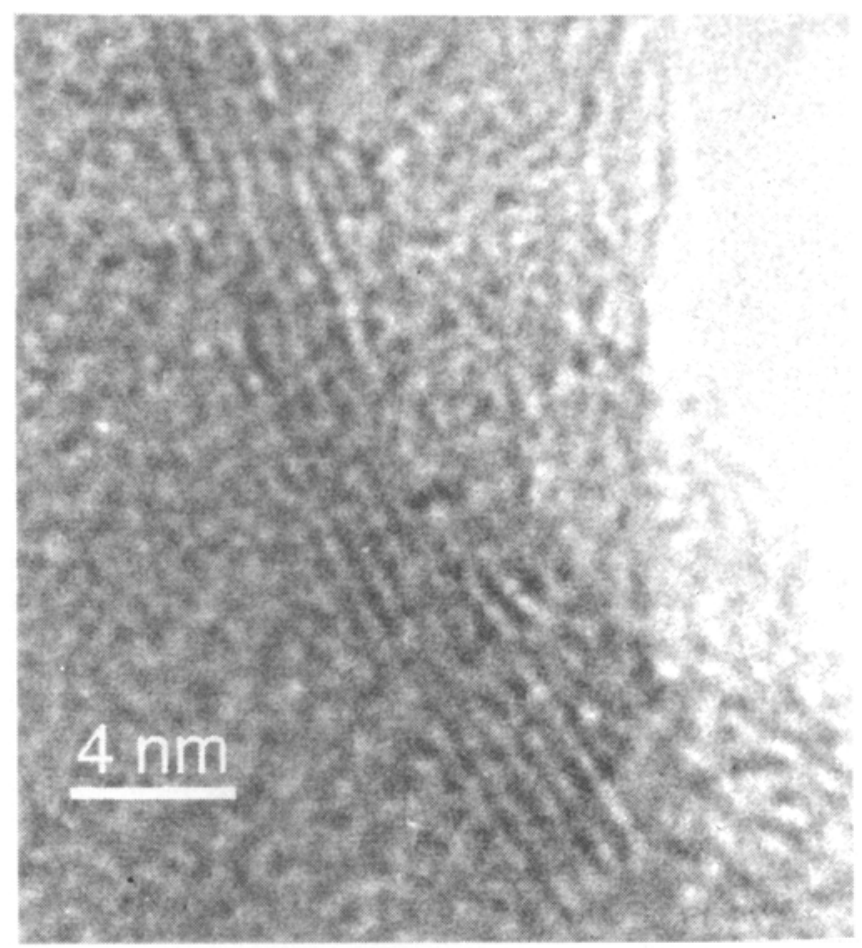

Figure 1. Transmission electron microscope (TEM) image shows the SWCNs which are slightly bent. The TEM image was taken after the AFI framework being removed using $\mathrm{HCl}$ acid, and the SWCNs being exposed in free space. coordinate diameter of the channels was $10 \cdot 1 \AA$ (inner diameter $7.3 \AA$ ), and the separation distance between two neighbouring channels was $13.7 \AA$. The AFI host was synthesized according to the method given in Qiu and Pan (1989). TPA molecules were encapsulated in the channels during crystal growth. The synthesis procedure of the SWCNs involved pyrolysis of TPA molecules in the AFI channels in a vacuum of $10^{-4}$ Torr at temperature $350 \sim 450^{\circ} \mathrm{C}$ and the formation of carbon nanotubes at $500 \sim 800^{\circ} \mathrm{C}$. The formation of the SWCNs was very sensitive to the quality of the AFI crystal, the type of hydrocarbon molecules in the AFI channels, and the pyrolysis conditions. The carbon-nanotube containing AFI crystal (C-AFI) behaved as a good polarizer with high absorption for the light polarized parallel to the channel direction $(E \| c)$ and with a high transparency for $E \perp c$, in consistent with the one-dimensional characteristics of the nanotubes.

\section{Results and discussion}

\subsection{Transmission electron microscopy observation}

As other zeolite crystal, the AFI framework is unstable under electron beam illumination. Also, in comparison

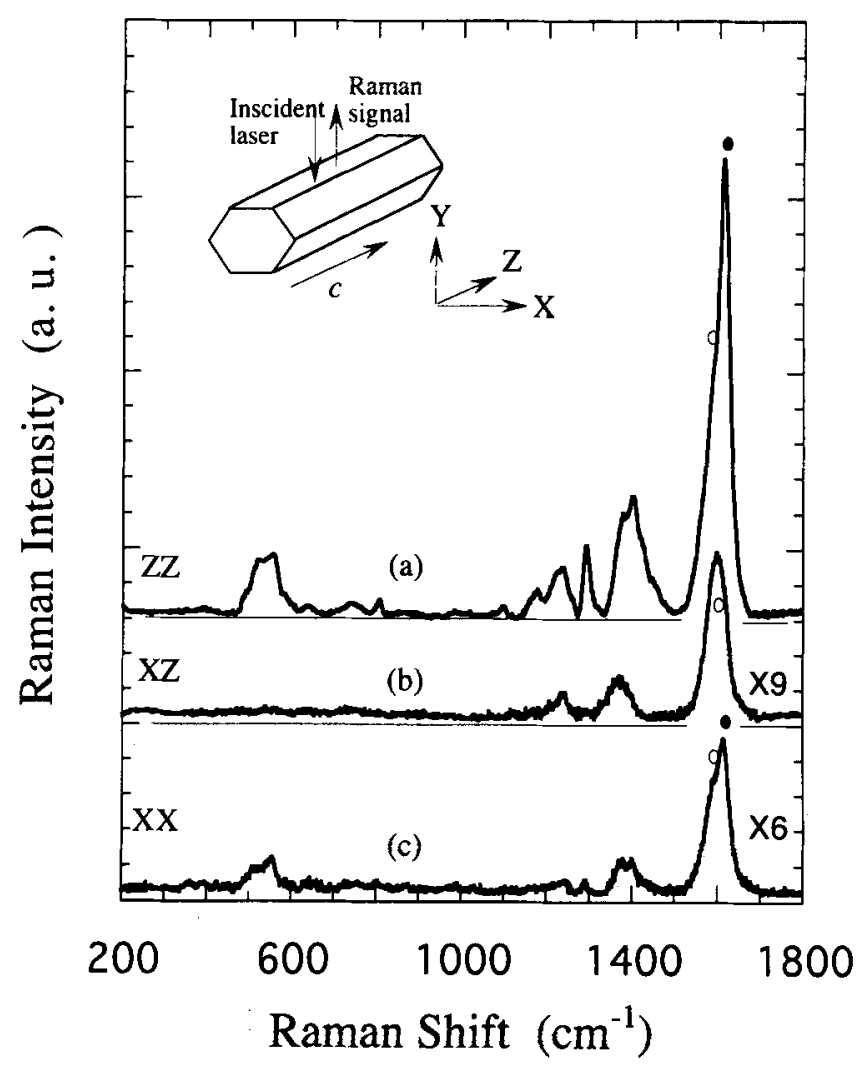

Figure 2. Polarized Raman spectra of SWCNs in three configurations: $Z Z, X Z$ and $X X$, where $Z$ and $X$ denote that the polarization of laser is along $Z$ or $X$-axis. The first (second) axis is the polarization direction of the incident laser (scattering light). The axis of the AFI crystal is along the $Z$-axis, as shown in the inset. 
with the heavy elements (Al, P, etc) of the AFI host, carbon element is too light to give a reasonable contrast in TEM image. Hence, it is hard to take a good TEM image for the carbon nanotubes accommodated in the AFI channels. In our experiment, we prepared the samples for TEM observations by removing the AFI host framework using acid etch, exposing the nanotubes into free space. Figure 1 shows the TEM image of the free-stand nanotubes. The image was taken using a Philips CM200 at $200 \mathrm{kV}$. Smoothly bent wire-like carbon nanotubes, with separation distance of about $7 \AA$, are seen in the image. Because of the small size of AFI channel, geometrically only single-walled carbon nanotubes can be expected in the channels. Hence, the wire-like TEM image can be attributed to the SWCN structure. The structure of the small tubes is stable when they are confined in the channels of AFI crystal, but they become unstable when they are exposed into free space especially under electron-beam irradiation. This fact indicates that the one-dimensional channels of AFI crystal play an important role in stabilizing small carbon nanotubes.

\subsection{Polarized Raman spectra}

It is difficult to determine the configuration of SWCNs accurately from electron microscopy. We can get more structural information from polarized Raman spectra for the nanotubes. Figure 2 shows the polarized Raman spectra of the SWCNs measured using $632.8 \mathrm{~nm}$ line of a He-Ne laser as excitation. Curves (a), (b) and (c) are for three different polarization configurations of $Z Z, X Z$ and $X X$, respectively, where $Z$ and $X$ denote the polarization directions defined in the inset of the figure. The first (second) notation is for the incident laser (scattering light). As seen in the figure, the Raman intensity measured in the $Z Z$ configuration is much stronger than those in other two configurations, indicating that the carbon specimen in the AFI channels are of one-dimensional characteristics. In the $Z Z$ configuration, a strong Raman signal at $1615 \mathrm{~cm}^{-1}$, with weak shoulders at low frequency side, dominates the spectrum. These Raman peak and shoulders have been attributed to the graphite-like longitudinal and transverse optical phonon modes branched off from the $\Gamma$ point to higher and lower energies with discrete wave vectors (Kasuya et al 1997). In low frequency region $\left(400 \sim 800 \mathrm{~cm}^{-1}\right)$, there is a significant Raman signal centred at $530 \mathrm{~cm}^{-1}$. Since the frequency is in the silent region for graphite and other carbon materials, this Raman signal can be assigned to the radial-breathing mode, which is special to the nanotube geometry. The breathing mode is not dependent on the chirality of nanotubes, but sensitive to the nanotube diameter (Jishi et al 1993; Saito et al 1998). The frequency of the mode is approximately a linear function of the reciprocal of the nanotube diameter. From the well-established data for $(10,10)$ armchair nanotubes
(Jishi et al 1993; Saito et al 1998), the frequency of the breathing mode can be estimated to be $448 \mathrm{~cm}^{-1}$ for the SWCNs in the AFI channels. The estimated Raman frequency is lower than the measured frequency of $530 \mathrm{~cm}^{-1}$ shown in figure 2 . The up-shift of frequency may result from the interaction between the nanotube wall and the rigid wall of channels of AFI crystals.

In the Raman spectrum of $Z Z$ configuration, some Raman bands are also seen in the intermediate frequency region $\left(1000 \sim 1500 \mathrm{~cm}^{-1}\right)$. In this region, the vibration frequency is expected to show the greatest chirality dependence, and the Raman-active modes are expected to show almost no intensity for nanotubes with relatively large diameters (Kasuya et al 1998). Hence the Raman signals observed in this frequency region in the $Z Z$ configuration (figure 2) might come from the symmetry lowering effect of the nanotubes. In fact, broad Raman peaks around $1350 \mathrm{~cm}^{-1}$ were observed in disordered graphite (Eklund et al 1995) and graphite nano-cluster (Kastner et al 1994) systems, and the ratio of the broad peak around $1350 \mathrm{~cm}^{-1}$ to the graphite line at $1582 \mathrm{~cm}$ is known to be sensitive to the symmetry-lowering effect (Tuinstra and Koenig 1970; Knight and White 1993).

As shown in figure 2 , the relative intensities of the Raman signals are sensitive to the polarization configurations. Because there exists ambiguity in the assignment of the Raman modes in the intermediate frequency region $\left(1000 \sim 1500 \mathrm{~cm}^{-1}\right)$, in the following we focus our discussion of the polarization properties to the breathing mode around $530 \mathrm{~cm}^{-1}$ and the graphite-like modes around $1600 \mathrm{~cm}^{-1}$. As seen in figure 2 , the breathing mode can be observed in the polarization configurations of $Z Z$ and $X X$, but disappeared in the depolarized spectrum of $X Z$ configuration. On the other hand, the Raman signal of the graphite-like modes peaked at $1615 \mathrm{~cm}^{-1}$ with a shoulder at $1585 \mathrm{~cm}^{-1}$ in the $Z Z$ configuration. This strong $1615 \mathrm{~cm}^{-1}$ line is replaced by a new line located at $1599 \mathrm{~cm}^{-1}$ in the $Z X$ configuration. While in the $X X$ configuration, $1615 \mathrm{~cm}^{-1}$ appears again but with a stronger shoulder at $1585 \mathrm{~cm}^{-1}$. The polarization dependence of these Raman lines can be explained well in terms of polarization selection rule of the Raman active modes for a zigzag nanotubes. Hence, we can conclude that the SWCN formed in the AFI channels is of zigzag symmetrical structure. Detailed analysis of the polarization properties of the Raman spectra is given elsewhere (Sun et al 1999).

\subsection{Electrical transport properties}

To easily handle the sample for electrical measurements, the sample was fixed inside a small hole drilled on a machinable ceramic using epoxy. Both sides of the sample were then polished until the hexagon ends of the crystal were exposed. Electrical contacts were made by evaporating a thin layer of gold on the two end 
surfaces. Current-voltage (I-V) properties were measured in the temperature range from $0.3 \mathrm{~K}$ to $300 \mathrm{~K}$. The inset of figure 3 shows the conductivity, $\sigma$ of the SWCNs measured near zero voltage plotted in logarithm scale as a function of the temperature, $T$. At room temperature the conductivity of the SWCN is in order of $10^{-1} \Omega^{-1} \mathrm{~cm}^{-1}$, which is lower than that reported for conductivity of metallic single-wall carbon nanotubes (Ebbesen et al 1996; Thess et al 1996; Yu Kasumov et al 1996). It is worth while to point out that the backbone of the AFI crystal with or without TPA in the channels is highly insulating. It contributes a current at least five orders of magnitude smaller than that of C-AFI samples. The conductivity of the SWCNs is monotonically decreased with decreasing temperatures, indicating that the SWCN is of semiconductor characteristics. The typical dc $I-V$ curve (open circles) measured at different temperatures is plotted in $\log -\log$ scale in figure 3 . The solid lines are fittings with $I \propto V$ and $I \propto V^{3 / 2}$ as indicated in the figure. The current starts out as a linear function of the voltage, but switches to a $V^{3 / 2}$ behaviour when the bias

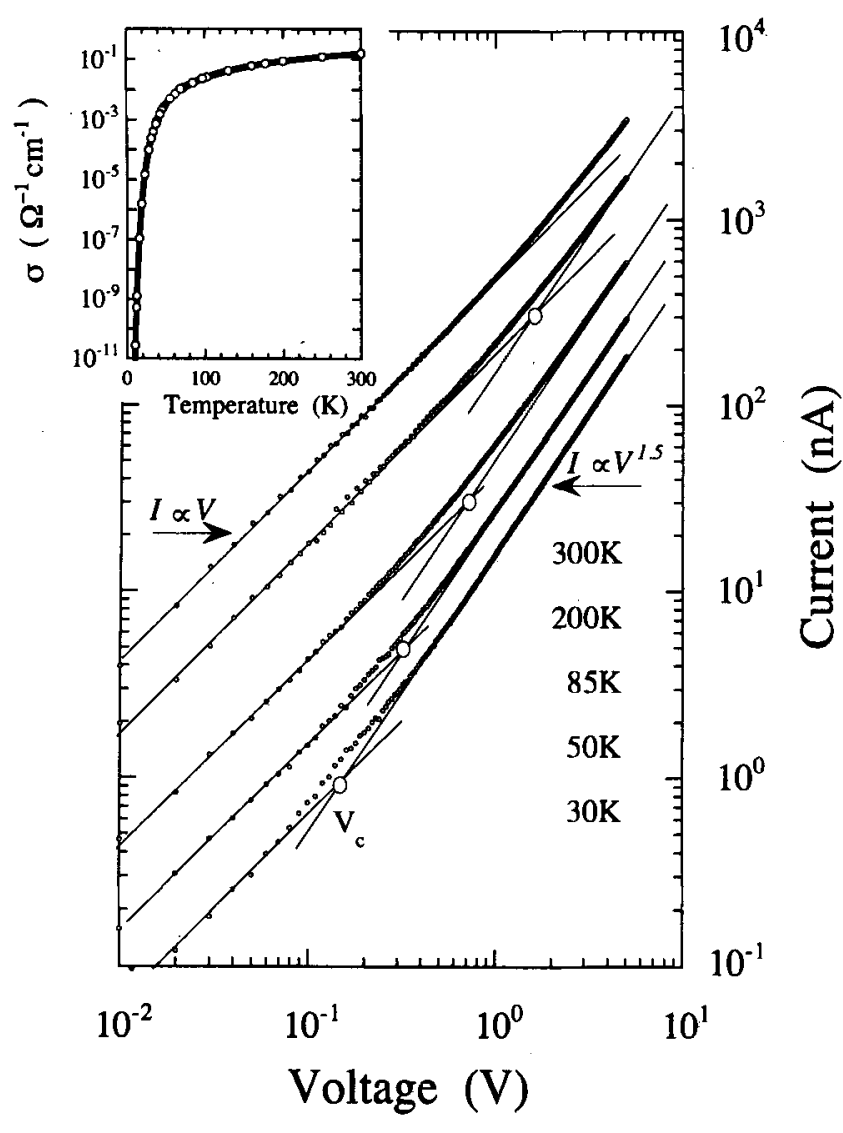

Figure 3. The experimental dc $I-V$ curves (open circles) measured at different temperatures and plotted on a log-log scale. The fittings shown as solid lines with $I \propto V$ in the low bias region and $I \propto V^{3 / 2}$ in the high bias region. The inset shows the conductivity measured near zero bias as a function of temperature. voltage is high or when the temperature is low. Since the SWCNs in our experiment are not intentionally doped, the conducting carriers are expected to be due to the thermally generated free carriers. Because the electrode metal and the SWCN have different work functions, a Schottky barrier can be formed at the lead-sample contact. When a bias voltage is applied, the barrier prevents one type of the thermally excited carriers (say electrons) to pass through, but allows the other type of carriers (say holes) to pass through freely. Hence, electrical conduction in the SWCN is expected to be a single-carrier process. A quantitative description of the experimental $I-V$ curves can then be obtained by evaluating the current across the sample by using the transport equation (Lampert et al 1964; Kao et al 1981)

$$
J=q \mu\left(n+n_{0}\right) E,
$$

and the Poisson equation

$$
\frac{l}{r} \frac{d\left(r^{2} E\right)}{\mathrm{d} r}=\frac{q n}{\varepsilon}
$$

here $q$ is the carrier charge, $\mu$ the carrier drift mobility, $E$ the applied electric field, $\varepsilon$ the static electric constant, $n$ and $n_{0}$ are the free carrier density injected from the electrode and the thermally generated carrier density, respectively. At high temperature or low bias voltage, $n_{0} \gg n$, the current is simply given by (1):

$$
I_{\Omega}=I_{\Omega 0} \mu\left(n+n_{0}\right) V,
$$

which is bulk-limited and is a linear function of bias voltage, $V$ (ohm current). At low temperature or under high bias, $n$ is comparable with $n_{0}$. Solving (1) and (2), yields $I_{\mathrm{sCL}}=I_{\mathrm{S} 0} \mu n_{0}^{1 / 2} V^{3 / 2}$, which is so-called spacecharge-limited (SCL) current. At a given temperature, the total current of the sample is due to the superposition of the $I_{\Omega}$ and the $I_{\mathrm{SCL}}$. We expect the current to be dominated by $I_{\Omega}$ at low bias and high temperature and by $I_{\text {SCL }}$ at high bias and low temperature. The solid lines in figure 3 show the calculated currents at different temperatures. The good agreement between experimental data and calculation also indicates that the SWCN confined in the AFI channel is an intrinsic semiconductor. The crossover voltage $V_{c}$, defined as the voltage at which $I_{\Omega}=I_{\mathrm{SCL}}$, is simply given by $V_{\mathrm{c}}=\left(I_{\Omega 0} / I_{\mathrm{S} 0}\right)^{2} n_{0}$, where $I_{\Omega 0} / I_{\mathrm{s} 0}$ is a constant for a given electrode contact configuration. It is interesting to notice that the $V_{\mathrm{c}}$ is linearly proportional to the thermal carrier density $n_{0}$, but is independent on the carrier mobility $\mu$. The simple dependence of $V_{c}$ on the carrier density $n_{0}$ offers us a ground to determine the band-gap for the SWCNs. As seen in figure 3 , the $V_{c}$ shifts towards higher value as increasing temperature, because of the increase of $n_{0}$ with the temperature. The $V_{c}$ measured by fitting the $I-V$ curves is plotted in figure 4 (open circles) as a 


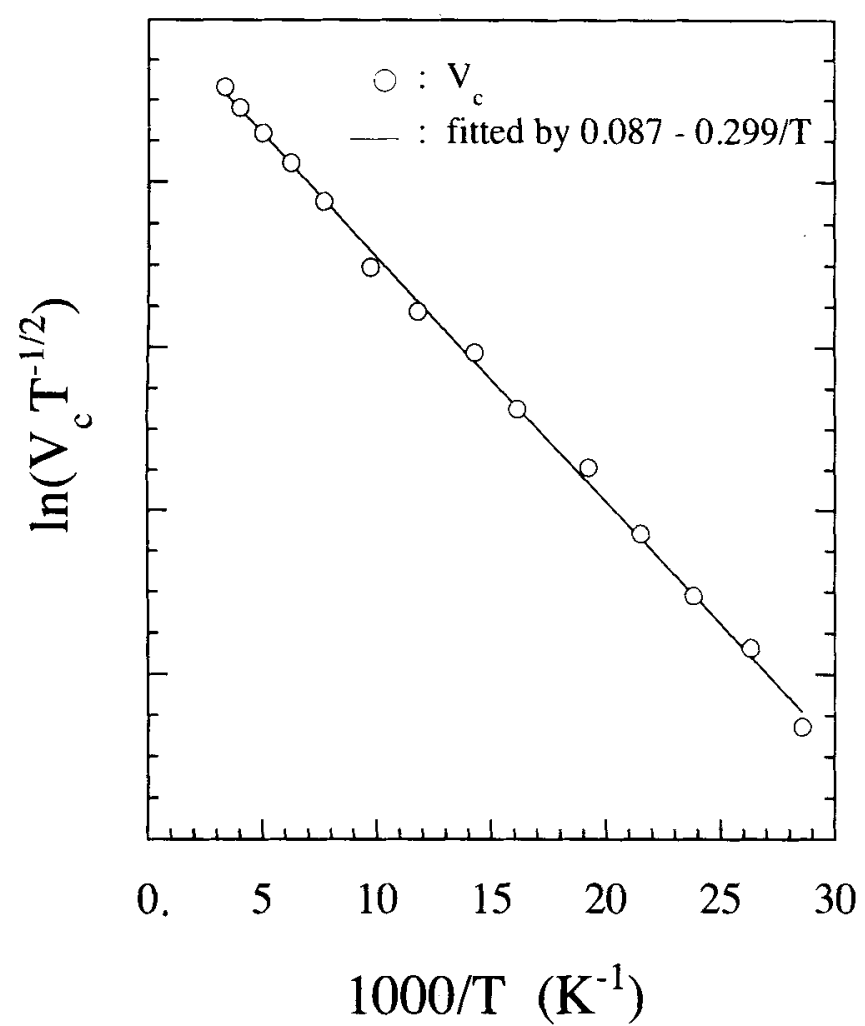

Figure 4. The temperature dependence of the crossover voltage, $V_{c}$ (solid circles), where $\ln \left(V_{c} T^{-1 / 2}\right)$ is plotted as a function of $1 / T$. The solid line is the temperature dependence of the thermal-generated carrier density calculated for the one-dimensional semiconductor.

function of the temperature. If we take the carbon nanotube as one-dimensional semiconductor and use the one-dimensional characteristic density-of-state $\left(E-E_{i}\right)^{-1 / 2}$, the temperature dependence of the thermal-generated carrier density $n_{0}$ can be calculated as

$$
n_{0}=A T^{1 / 2} \exp \left(-E_{\mathrm{g}} / 2 k_{\mathrm{B}} T\right),
$$

here $A$ is a constant, $E_{\mathrm{g}}$ the band-gap energy, $k_{\mathrm{B}}$ the Boltzman constant and $T$ the temperature. The solid curve in figure 4 is the plot $\ln \left(n_{0} / T^{1 / 2}\right)$ against $1 / T$, which gives a linear line. The band-gap energy $E_{g}$ can then be determined from the line slope. This relationship is shown in figure 4 . The experimental data $V_{\mathrm{c}}$ (open circles) is fitted well using a line slope of $-299 \mathrm{~K}$ which corresponds to an energy gap of $52 \mathrm{meV}$.

In summary, we have fabricated mono-sized SWCNs with well-defined structure symmetry. They were characterized through TEM, Raman scattering and electrical transport measurements. Our results would open a door to further detailed studies on the intrinsic properties of semiconducting carbon nanotubes, presently under way.

\section{Acknowledgements}

We are grateful to Professors L L Chang, M M T Loy, $P$ Sheng and G K L Wong for their encouragement and valuable comments. In particular, (HDS) is grateful to Prof. G K L Wong for introducing him to this research area. The authors also thank $\operatorname{Dr} \mathrm{C} T$ Chen for his theoretical advice and useful discussion. The TEM image was taken by Dr $\mathrm{N}$ Wang. This research was supported by the RGC Committee of Hong Kong, and the EHIA program from HKUST. (JC) and (GL) would like to acknowledge the support from the National Natural Science Foundation of China.

\section{References}

Amelincks S et al 1994 Science 265635

Blase X, Benedict L X, Shirley E L and Louie S G 1994 Phys. Rev. Lett. 721878

Bockrath M et al 1997 Science 2751922

Dresselhaus M S, Dresselhaus D and Saito R 1995 Carbon 33883

Dresselhaus M S, Dresselhaus G and Eklund P C 1996 Science of fullerenes and carbon nanotubes (New York: Academic Press)

Ebbesen T W, Lezec H J, Hiura H, Bennet J W, Ghaemi H F and Thio T 1996 Nature 38254

Eklund P C, Holden J M and Jishi R A 1995 Carbon 33959

Guo T, Nikolaev P, Thess A, Colbert D T and Smalley R E 1995 Chem. Phys. Lett. 24349

Iijima S 1991 Nature 35456

Jishi R A, Venkataraman L, Dresselhaus M S and Dresselhaus G 1993 Chem. Phys. Lett. 20977

Journet $C$ et al 1997 Nature 388756

Kao K C and Hwang W 1981 Electrical transport in solids (London: Pergamon Press Ltd)

Kastner J et al 1994 Chem. Phys. Lett. 22153

Kasumov A V, Khodos I I, Ajayan P M and Aolliex C 1996 Europhys. Lett. 34429

Kasuya A et al 1997 Phys. Rev. Lett. 784434

Kasuya A et al 1998 Phys. Rev. B57 4999

Knight D S and White W B 1993 J. Mater. Res. 4385

Kukovitsukii E F, Chemozatonskii A, L'vov S G and Mel'nik N N 1997 Chem. Phys. Lett. 266323

Lampert M A, Many A and Mark P 1964 Phy. Rev. 135 Al444

Langer L et al 1996 Phys. Rev. Lett. 76479

Lee R S, Kim H J, Fischer J E, Thess A and Smalley R E 1997 Nature 388255

Mintmire J W and White C T 1995 Carbon 33893

Qiu S and Pan W 1989 Zeolites 9440

Saito R, Takeya T, Kimura T, Dresselhaus $G$ and Dresselhaus M S 1998 Phys. Rev. B57 4145

Sun H D, Tang Z K, Chen J and Li G 1999 Solid State Commun. (in press)

Tans S J et al 1997 Nature 386474

Thess A et al 1996 Science 273483

Tuinstra F and Koenig J L 1970 J. Chem. Phys. 531126

Yu Kasumov A, Khodos I I, Ajayan P M and Colliex C 1996 Europhys. Lett. 34429 